\title{
Design and Analysis of Network Disk System Based on Web
}

\author{
Bin Liu, Yan Zhang and Mian Yi \\ Jiangxi Technical College of Manufacturing, Department of Information Engineering
}

\author{
Keywords: Cloud storage; Network disk; File system
}

\begin{abstract}
There is extensive demand for mobile office and storage network disk system for enterprise network and campus network on the market because traditional network disk technology has various defects in performance, sharing between users, safety and scalability. Targeting at these defects, this paper proposes a high-performance network disk system framework based on cloud storage and adopts the distributive file system MooseFS to realize the cluster framework to store and access users' data. In terms of security, SAMBA is combined to manage users' right and users' data storage supports the encryption of 128 bit AES, guaranteeing the security of the transmission lines. Finally, this paper proposes the synchronization mode based on Web-access and client while combining users' demands. The results show that the system has obvious advantages in performance, security and scalability, etc.
\end{abstract}

\section{Introduction}

With the rapid development of cloud storage, there are richer and richer network disk products for the public, such as Dropbox, Google Drive, SkyDrive, Aliyun and Grandcloud, etc., which have also attracted an increasingly number of users to choose to store their personal information on the network disk. Network disk client is a professional tool to assist users to upload, download and synchronize local files to the service client. When users need to synchronize the local files to the service client, the client end will automatically upload the entire local files to server through the standard connection portfolio. While in reality, there are only partial differences between the files to be uploaded to the server and existing files in server, and if the entire files are uploaded synchronously, a lot of bandwidth will be wasted. Especially in the current ADSL technology, the uploading bandwidth only accounts for $15 \%$ of the download bandwidth, and uploading a huge amount of repeated files will instantly increase the delay in time of operating files synchronously and reduce users' experience. However, due to the restriction of the ADSL technology, uploading speed is usually slower than the downloading speed, which greatly restricts the network disk's performance of synchronizing files to the server and affects network disk users' experience.

This paper proposes a network disk system design method based on Web, which adopts the MySOL as the backstage database and JSP as the front interface to develop program. The designed system can be applied conveniently without subject to the restriction of the operation system and platform and adopts certain measures to process possible safety issues.

\section{Network Disk}

Network hardware (network disk in short) is an online storage service based on users' space, on which users can upload, download and share data through the Internet. There is extensive demand for mobile office and storage network disk system for the enterprise network or campus network on the market. With the constant development of the storage technology, it is obvious that traditional technology is unable to cope with such vast storage demand with various bottlenecks such as slow transmission speed, disastrous backup and recovery ability, poor security and high operation costs. The recently applied cloud storage technology has brought new revolution to the network disk industry.

Cloud storage is a data center constructed on the high-speed distributive storage network. It integrates a huge amount of various types of storage equipment on the Internet through application software to form safe data storage and access system, so it is applicable to various large, 
medium-sized and small enterprises and individual users to satisfy their demand for storing, backup and file data and materials, etc. In recent years, classic cloud storage service products both in China and abroad include iCloud, Cloud Drive, Dropbox, SkyDrive and Amazon S3, etc. Ever since 2009, cloud storage has been vigorously developing and promoted in China and each famous enterprise like Lenovo, Sino, Huawei, Kingsoft, Qihu 360 and Tencent has promoted their corresponding cloud storage products or service. Tsinghua University has also promoted the cloud storage service (Corsair and Meepo) for its internal users.

\section{Database Design}

Network disk is a distant file operation system which must have simple functions of normal file systems, such as input, and reading, etc. In addition, it must also have corresponding certified connection and management over users. According to the above analysis, framework diagram of this network disk system is as shown in Fig. 1.

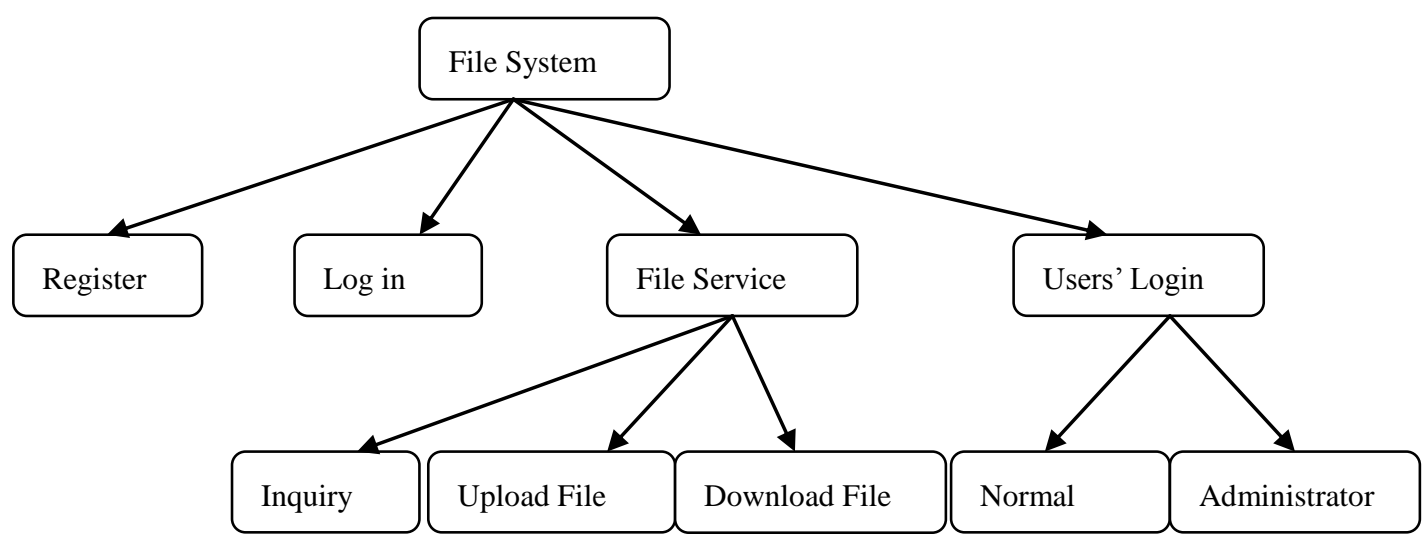

Figure 1. Functional Structure of the System

MySOL database is adopted in this system and the corresponding database tables are a files table of the file information and the users table of user information, which are realized with the Java language that can prevent the injection of SQL. The simplest way is to avoid SQL from joining together. SQL can attack the injection because new logic is added to the original SQL language. If Prepared Statement is used to replace Statement to implement the SQL language and add parameters later, SQL will fail to attack injection because Prepared Statement cannot allow different injection times to change the logic structure of inquiry, and most SQL injections have been blocked. In addition, users' input can be screened at the WEB level to prevent SQL injection, such as using Filter to filter the overall form parameters.

\section{Distributive File System and Cluster Framework}

Distributive file system is the core of cloud storage technology, and through the distributive file system, large-scaled, safe and reliable file systems can be realized to support the large-scale expansion of the system. At present, widely applied file systems include the Sun corporate's Lustre, Hadoop framework's HDFS, MogileFS, DastDFS and MooseFS, etc. These distributive file systems have their own characteristics, so they cannot be applied universally but can be only applied to special application scene. For example, HDFS is mainly applied to processing applicable program of huge data set. MooseFS is a free open-source distributive file system, which is a universal file system that can be applied without modifying the upper application. Compared with other distributive file systems, MooseFS has the following advantages:

- Files are divided into different blocks and storage at different data nodes, and there is no risk for the default hardware to leak data;

- Online expansion is allowed and the system's framework is very flexible; 
- Simple deployment;

- System framework is highly available without failure to all the components;

- File objects are highly available and file redundancy can be set;

- Web GUI monitoring connector can be provided ;

- Improve the efficiency of random read or write;

- More importantly, it can improve the efficiency of small-capacity files' read and write,

It is applicable to the random file storage or withdrawal of enterprise network or campus network users. MooseFS is selected as the basic framework of distributive cluster while combing the application background, which is as shown in Fig. 2:

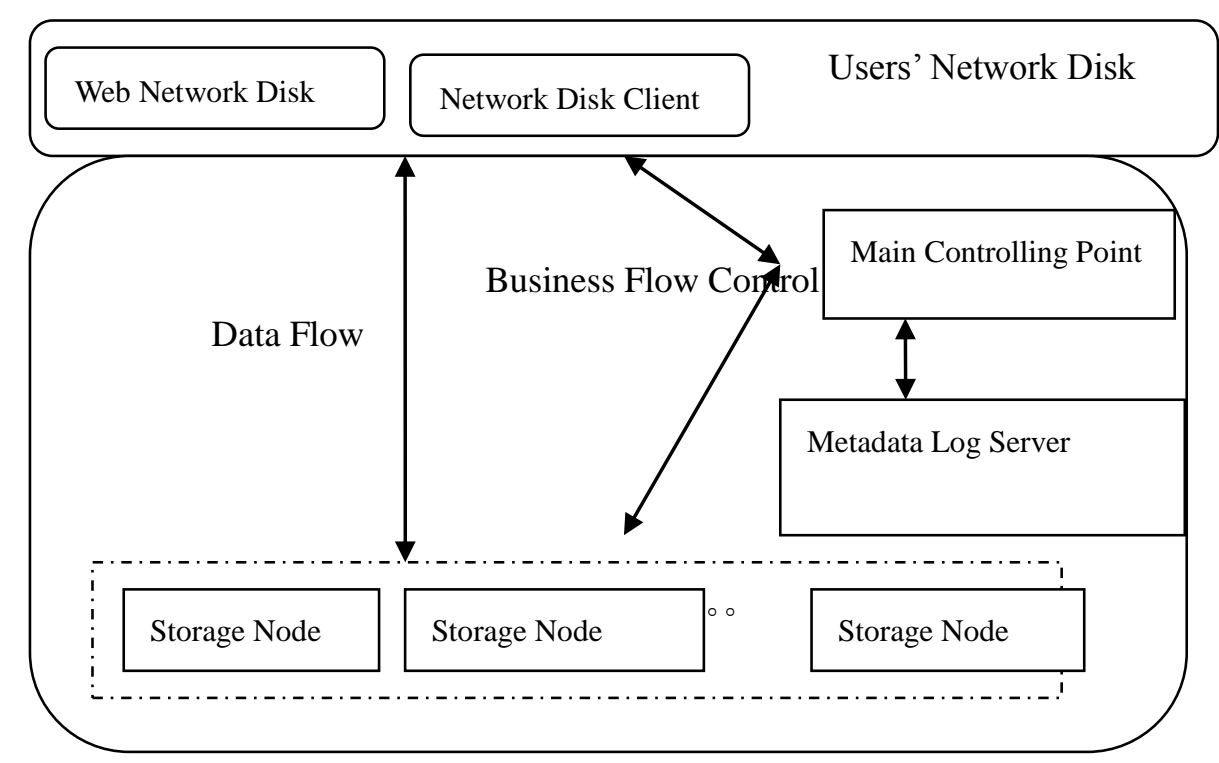

Figure 2. MooseFS and Cluster Framework

When users access the network disk space, they first send request to the main controlling point, which will designate a storage node server to interact with users' data according to the dynamics of the storage nodes' loading. Therefore, business flow is obtained through accessing the main controlling point, and data flow is obtained through accessing storage node. Such separation of business flow and controlling flow greatly improves the efficiency of storing and withdrawing data.

\section{Conclusion}

Network disk is a convenient and rapid file application system that can reduce individual users' load of managing files and investment of corresponding construction resources. Ordinary users can access this system through the browser and manage files, and administrator manages users' information and gather their data. The system also adopts necessary safety measures to guarantee the security of users' data. After being tested, this system can effectively allow users to monitor management over data in a distance in a reliable and safe environment. Design in this paper realizes a network disk system based on Web, so files can be stored and managed through ordinary browser. Therefore, such design is very practical. The system also conducts targeted processing in security to guarantee the security of users' data.

\section{References}

[1] Katz R H, Gibson G A, Patterson D A. Disk System Architectures for High Performance Computing[J]. Proceedings of the IEEE, 1989, 77(12):1842-1858. 
[2] Setiawan J, Henning T, Launhardt R, et al. A young massive planet in a star-disk system.[J]. Nature, 2008, 451(7174):38-41.

[3] Mori, K., Hirai, H., Otsuki, H., Takahashi, T., Naruse, J., \& Nishimura, Y., et al. (1993). Disk system with sub-actuators for fine head displacement. US, US 5189578 A.

[4] Comerford, Liam D., and S. R. White. "Hardware key-on-disk system for copy-protecting magnetic storage media." US, US4577289. 1986.

[5] Kaneda, Y., Oeda, T., Honda, K., Matsunami, N., Akiyama, H., \& Arakawa, H., et al. (1999). Array type disk system updating redundant data asynchronously with data access. US, US5958067.

[6] Kamo Y, Kakuta H, Tanaka A, et al. Disk system and power-on sequence for the same: US, US 6397294 B2[P]. 2002.

[7] Sridharan T K, Williams S J, Fuller G A. Direct Detection of a (Proto)Binary-Disk System in IRAS 20126+4104[J]. Astrophysical Journal, 2005, 631(1):L73-L76.

[8] Baylor S J, Corbett P F, Fitch B G, et al. Using virtual disks for disk system checkpointing: US, US 5634096 A[P]. 1997.

[9] Slaughter G L, Traversat B A, Herndon R, et al. Highly available cluster virtual disk system: CA, US5964886[P]. 1999.

[10]Dias D M, Mukherjee R. Real-time shared disk system for computer clusters: US, US 6182197 B1[P]. 2001.

[11]Chen, Ben Wei, Y. Chien, and C. T. Tang. "Secure and scalable solid state disk system." US, US8527781. 2013.

[12]Pharris, Kenton J., and D. L. Schell. "Method and apparatus for calibrating an optical disk system." US, US4907212. 1990. 